\title{
Utilizing Machine Learning for Pre- and Postoperative Assessment of Patients Undergoing Resection for BCLC-0, $A$ and B Hepatocellular Carcinoma: Implications for Resection Beyond the BCLC Guidelines
}

\author{
Diamantis I. Tsilimigras, MD ${ }^{1}$, Rittal Mehta, MPH ${ }^{1}$, Dimitrios Moris, MD, PhD ${ }^{1}$, Kota Sahara, MD ${ }^{1}$, \\ Fabio Bagante, $\mathrm{MD}^{1,2}$, Anghela Z. Paredes, MD, MS ${ }^{1}$, Ayesha Farooq, MD ${ }^{1}$, Francesca Ratti, $\mathrm{MD}^{3}$, \\ Hugo P. Marques, $\mathrm{MD}^{4}$, Silvia Silva, $\mathrm{MD}^{4}$, Olivier Soubrane, $\mathrm{MD}^{5}$, Vincent Lam, $\mathrm{MD}^{6}$, George A. Poultsides, $\mathrm{MD}^{7}$, \\ Irinel Popescu, $\mathrm{MD}^{8}$, Razvan Grigorie, $\mathrm{MD} \mathrm{PhD}^{8}$, Sorin Alexandrescu, $\mathrm{MD}^{8}$, Guillaume Martel, $\mathrm{MD}^{9}$, \\ Aklile Workneh, $\mathrm{MD}^{9}$, Alfredo Guglielmi, $\mathrm{MD}^{2}$, Tom Hugh, $\mathrm{MD}^{10}$, Luca Aldrighetti, $\mathrm{MD}^{3}$, \\ Itaru Endo, MD, PhD ${ }^{11}$, and Timothy M. Pawlik, MD, MPH, PhD, FACS, FRACS (Hon.) ${ }^{1}$ \\ ${ }^{1}$ Department of Surgery, The Ohio State University Wexner Medical Center, Columbus, OH; ${ }^{2}$ Department of Surgery, \\ University of Verona, Verona, Italy; ${ }^{3}$ Department of Surgery, Ospedale San Raffaele, Milan, Italy; ${ }^{4}$ Department of \\ Surgery, Curry Cabral Hospital, Lisbon, Portugal; ${ }^{5}$ Department of Hepatobiliopancreatic Surgery, APHP, Beaujon \\ Hospital, Clichy, France; ${ }^{6}$ Department of Surgery, Westmead Hospital, Sydney, NSW, Australia; ${ }^{7}$ Department of Surgery, \\ Stanford University, Stanford, CA; ${ }^{8}$ Department of Surgery, Fundeni Clinical Institute, Bucharest, Romania; ${ }^{9}$ Department \\ of Surgery, University of Ottawa, Ottawa, ON, Canada; ${ }^{10}$ Department of Surgery, The University of Sydney, School of \\ Medicine, Sydney, NSW, Australia; ${ }^{11}$ Yokohama City University School of Medicine, Yokohama, Japan
}

\begin{abstract}
Background. There is an ongoing debate about expanding the resection criteria for hepatocellular carcinoma (HCC) beyond the Barcelona Clinic Liver Cancer (BCLC) guidelines. We sought to determine the factors that held the most prognostic weight in the pre- and postoperative setting for each BCLC stage by applying a machine learning method.

Methods. Patients who underwent resection for BCLC-0, A and B HCC between 2000 and 2017 were identified from an international multi-institutional database. A Classification and Regression Tree (CART) model was used to generate homogeneous groups of patients relative to
\end{abstract}

Electronic supplementary material The online version of this article (https://doi.org/10.1245/s10434-019-08025-z) contains supplementary material, which is available to authorized users.

(C) Society of Surgical Oncology 2019

First Received: 3 July 2019;

Published Online: 6 November 2019

T. M. Pawlik, MD, MPH, PhD, FACS, FRACS (Hon.)

e-mail: tim.pawlik@osumc.edu overall survival (OS) based on pre- and postoperative factors.

Results. Among 976 patients, 63 (6.5\%) had BCLC-0, 745 (76.3\%) had BCLC-A, and 168 (17.2\%) had BCLC-B HCC. Five-year OS among BCLC-0/A and BCLC-B patients was $64.2 \%$ versus $50.2 \%$, respectively ( $p=0.011$ ). The preoperative CART model selected $\alpha$-fetoprotein (AFP) and Charlson comorbidity score (CCS) as the first and second most important preoperative factors of OS among BCLC-0/A patients, whereas radiologic tumor burden score (TBS) was the best predictor of OS among BCLC-B patients. The postoperative CART model revealed lymphovascular invasion as the best postoperative predictor of OS among BCLC-0/A patients, whereas TBS remained the best predictor of long-term outcomes among BCLC-B patients in the postoperative setting. On multivariable analysis, pathologic TBS independently predicted worse OS among BCLC-0/A (hazard ratio [HR] 1.04, 95\% confidence interval [CI] 1.02-1.07) and BCLC-B patients (HR 1.13, 95\% CI 1.06-1.19) undergoing resection.

Conclusion. Prognostic stratification of patients undergoing resection for $\mathrm{HCC}$ within and beyond the BCLC resection criteria should include assessment of AFP and 
comorbidities for BCLC-0/A patients, as well as tumor burden for BCLC-B patients.

Hepatocellular carcinoma (HCC) ranks as the fifth most common cancer worldwide and is considered the most rapidly increasing cause of cancer-related deaths in the US. ${ }^{1,2}$ Accurate preoperative staging is critical to define the prognosis of patients with $\mathrm{HCC}$ and inform treatment recommendations. ${ }^{3,4}$ Among the staging systems available, the Barcelona Clinic Liver Cancer (BCLC) classification has been largely adopted and routinely used by physicians in the West. ${ }^{5-7}$ According to the BCLC system, liver resection should be offered only to patients with very early (i.e. BCLC-0) or early (i.e. BCLC-A) stage HCC, whereas patients with intermediate and advanced HCC (i.e. BCLC$\mathrm{B}$ and BCLC-C) should be referred for non-surgical management, including transarterial chemoembolization (TACE) and sorafenib, respectively. ${ }^{6,7}$

Advances in surgical techniques, radiologic assessment, and perioperative management have increased the resectability rates of $\mathrm{HCC}$ worldwide. Indeed, more than $60 \%$ of patients diagnosed with HCC are currently offered surgery in Asia, whereas the corresponding rates in the West range from 25 to $40 \% .^{8,9}$ Of note, a growing number of patients currently undergo surgery for HCC beyond the BCLC criteria, with acceptable reported outcomes. ${ }^{8}$ For example, Wada et al. reported a 5-year overall survival (OS) of $63.4 \%$ among 85 patients with multinodular BCLC-B HCC undergoing liver resection. ${ }^{10}$ In a separate study, a select group of patients with BCLC-B or -C HCC had improved long-term outcomes following resection versus locoregional therapy or best supportive care. ${ }^{11}$ Nevertheless, while benefitting certain individuals, surgery does not provide a benefit to all patients. In turn, surgeons need to weigh the benefits of operating on patients beyond the current BCLC criteria against the possible risks associated with more extensive liver resection.

Given the ongoing debate around expanding the resection criteria for $\mathrm{HCC}$, defining the most important clinicopathologic factors relative to long-term outcomes is important to help construct a tailored therapeutic strategy based on individual patient characteristics. As such, the objective of the current study was to identify the factors most predictive of survival prior to and following resection of HCC within and beyond the BCLC guidelines. Specifically, we sought to determine the factors that held the most prognostic weight in the pre- and postoperative setting for each BCLC stage by applying a machine learning method on a large multi-institutional cohort of patients who underwent curative-intent resection of HCC.

\section{METHODS}

\section{Study Population and Data Collection}

Patients who underwent resection for HCC between 2000 and 2017 were identified from an international multiinstitutional database. Patients were treated at 1 of 11 participating institutions: The Ohio State University Wexner Medical Center, Columbus, OH, USA; Yokohama City University School of Medicine, Yokohama, Japan; University of Verona, Verona, Italy; Ospedale San Raffaele, Milan, Italy; Curry Cabral Hospital, Lisbon, Portugal; APHP, Beaujon Hospital, Clichy, France; Westmead Hospital, Sydney, NSW Australia; Stanford University, Stanford, CA, USA; Fundeni Clinical Institute, Bucharest, Romania; University of Ottawa, Ottawa, ON, Canada; and The University of Sydney, School of Medicine, Sydney, NSW, Australia. Patients were followed and outcomes were recorded in a prospectively maintained multi-institutional database. Patients with BCLC stages 0, $\mathrm{A}$ and $\mathrm{B} \mathrm{HCC}^{6}$ were included in the final cohort. Patients who (1) had BCLC-C tumors, (2) did not receive curative intent resection, (3) did not have data on pathologic as well as radiologic tumor size and number, and, finally (4) did not have adequate follow-up data were excluded from the analysis. The study was approved by the Institutional Review Boards of all participating institutions.

Demographic and clinical data included age, sex, American Society of Anesthesiologists (ASA) score, Charlson Comorbidity Index (CCI) score, history of cirrhosis, hepatitis $\mathrm{B}$ virus (HBV)/hepatitis $\mathrm{C}$ virus $(\mathrm{HCV})$ infection, laboratory values (i.e. platelet count, albumin, total bilirubin, aspartate aminotransferase [AST], alanine aminotransferase [ALT], international normalized ratio [INR], $\alpha$-fetoprotein [AFP]), Child-Pugh class, BCLC stage, minimally invasive surgery, type of surgical resection (i.e. minor or major), tumor size and grade, pathologic lymphovascular invasion, liver capsule involvement, margin status (i.e. R0, R1, R2), as well as radiologic and pathologic tumor burden score (TBS).

\section{Definitions}

According to the latest European Association for the Study of Liver (EASL) guidelines, BCLC-0 was defined as a single tumor $<2 \mathrm{~cm}$; BCLC-A was defined as a single tumor $\geq 2 \mathrm{~cm}$ or two to three nodules, all $<3 \mathrm{~cm}$; and BCLC-B was defined as two to three nodules $\geq 3 \mathrm{~cm}$ or four or more nodules. ${ }^{6}$ In case of multiple nodules, tumor size was calculated on the basis of largest lesion size. Major hepatectomy was defined as resection of three or more Couinaud segments, ${ }^{12}$ and TBS was defined as the distance from the origin of a Cartesian plane, and 
comprised of two variables: maximum tumor size ( $x$-axis) and number of tumors ( $y$-axis), so that $\mathrm{TBS}^{2}=$ (maximum

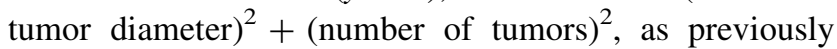
described. ${ }^{13}$ Pathologic TBS for each patient was calculated based on final pathologic reports, while radiologic TBS was calculated based on the information obtained from preoperative computed tomography scans.

\section{Statistical Analysis}

Descriptive statistics were presented as median (interquartile range $[\mathrm{IQR}]$ ) and frequency (\%) for continuous and categorical variables, respectively. OS was defined as the time interval between the date of hepatectomy and the date of death. For patients who remained alive, OS was censored at the date of last follow-up. Recurrence-free survival (RFS) was defined as the time interval between hepatectomy and date of recurrence. Bivariate survival analyses were performed using the log-rank test and presented using Kaplan-Meier curves. Variables that were significant on bivariate analysis $(p<0.05)$ and did not violate the proportional hazards assumptions were entered into the multivariable Cox regression model. A backward step selection method was used to eliminate non-significant variables using a $p$ value $<0.10$. The level of statistical significance for all tests was set at $\alpha=0.05$. All statistical analyses were performed using SPSS version 25 (IBM Corporation, Armonk, NY, USA).

\section{Classification and Regression Tree (CART) Model}

A Classification and Regression Tree (CART) model was used to generate homogeneous groups of patients relative to OS based on pre- (preoperative CART model) and postoperative (preoperative CART model) factors. CART is a class of nonparametric risk prediction models that performs a recursive partition of the 'covariate space', as previously described. ${ }^{14}$ Survival regression trees are able to create an easy-to-interpret prediction model that identifies the optimal cut-off values and stratifies patients into different groups relative to survival. ${ }^{14}$ The 'goodness of fit' method was used to maximize between-node separation, and the log-rank statistic was used to measure betweennode heterogeneity. The optimal cut-off was based on best split among the variable using the highest log-rank statistic. To prune the tree and to minimize overfitting, the lowest complexity parameter was used with the one minus standard error rule that reflected the trade-off between the tree complexity and how well the tree fit the data. To assess the predictive performance of the final model, the concordance index (c-index) for time-to-event data was calculated with the bootstrapping resample method $(n=2000)$ using the $\mathrm{R}$ CRAN package Hmisc. The CART model was developed using the R CRAN software for statistical computing version 3.6.0 with the additional packages: survival, partykit, rpart, Hmisc, caret and ROCR.

\section{RESULTS}

\section{Baseline Characteristics in the Entire Cohort}

Among 976 patients with HCC included in the final analytic cohort, $6.5 \%(n=63)$ of patients had BCLC- 0 , $76.3 \%(n=745)$ had BCLC-A, and $17.2 \%(n=168)$ had BCLC-B HCC (Table 1). Median patient age was 67 years (IQR 59-74), most patients were male $(n=748,76.7 \%)$, had an ASA score $\leq 2(n=570,63.1 \%)$, and a Charlson comorbidity score $(\mathrm{CCS}) \leq 4(n=489,54.2 \%)$. History of cirrhosis and $\mathrm{HBV}$ and $\mathrm{HCV}$ infection were present in $38.9 \%(n=379), 27.6 \%(n=267)$ and $31.3 \%(n=303)$ of patients, respectively. The vast majority of patients had AFP $\leq 400 \mathrm{ng} / \mathrm{mL}(n=694,80.5 \%)$ and Child-Pugh class A liver function $(n=699,95.2 \%)$. Approximately onequarter of patients underwent minimally invasive surgery $(n=237,24.4 \%)$ and approximately one-third had a major resection $(n=334,35.1 \%)$. Median tumor size was $5 \mathrm{~cm}$ (IQR 3.0-8.5) and most tumors were well-to-moderately differentiated $(n=753,79.9 \%)$. Median pathologic TBS was $5.1 \mathrm{~cm}$ (IQR 3.4-8.8), and radiologic TBS was comparable (median $5.1 \mathrm{~cm}$ [IQR 3.3-8.5]). Following resection, lymphovascular invasion and liver capsule involvement were present in 39.2\% $(n=346)$ and $32.8 \%$ $(n=240)$ of tumor specimens, respectively. The vast majority of patients had an R0 resection $(n=825,86.8 \%)$.

\section{Preoperative CART Model: Selection of Patients for Resection Within and Beyond the Barcelona Clinic Liver Cancer (BCLC) Guidelines}

CART analysis revealed that AFP levels, CCS, and radiologic TBS were the best preoperative prognostic factors associated with OS among BCLC-0/A and BCLC-B patients undergoing resection (Fig. 1). Of note, 5-year OS among BCLC-0/A and BCLC-B patients undergoing resection was $64.2 \%$ versus $50.2 \%(p=0.011)$, respectively (electronic supplementary Fig. 1). Five-year RFS among patients undergoing resection for BCLC-0/A and BCLC-B HCC was $36.9 \%$ versus $24.5 \%$, respectively $(p<0.001)$. Among BCLC-0/A patients, CART selected AFP and CCS as the first and second most important preoperative factors associated with OS. Specifically, patients with lower AFP levels and CCS $\leq 4$ had a 5 -year OS as high as $75 \%$, whereas patients with the highest AFP levels had only a 5 -year OS of $23.6 \%$. Of note, among BCLC-B patients, only radiologic TBS was selected by the CART 
TABLE 1 Demographics and patient characteristics in the entire cohort $(N=976)$

\begin{tabular}{l} 
Variable \\
\hline Age, years [median (IQR)] \\
Sex \\
Male \\
Female \\
ASA-PS \\
$\leq 2$ \\
$>2$
\end{tabular}

Charlson comorbidity score

$\leq 4$
$>4$

Cirrhosis

No

Yes

HBV infection

No

Yes

HCV infection

No

Yes

Platelet count, $\times 10^{3} / \mu \mathrm{L}$

$\leq 150$

$>150$

Albumin, g/dL

$\leq 3.5$

$>3.5$

Total bilirubin, mg/dL

$\leq 1.2$

$>1.2$

AST, U/L

$\leq 40$

$>40$

ALT, U/L

$\leq 56$

$>56$

PT/INR

$\leq 1.1$

$>1.1$

AFP, ng/mL

$\leq 400$

$>400$

Child-Pugh classification

A

B

BCLC staging classification

0

A

B

$177(22.8)$

$601(77.2)$

$760(84.3)$

142 (15.7)

$391(44.4)$

$490(55.6)$

591 (64.6)

324 (35.4)

709 (77.5)

$206(22.5)$

$694(80.5)$

168 (19.5)

699 (95.2)

35 (4.8)

$63(6.5)$

745 (76.3)

168 (17.2)
TABLE 1 (continued)

\begin{tabular}{lc}
\hline Variable & Value \\
\hline Minimally invasive surgery & $735(75.6)$ \\
No & $237(24.4)$ \\
Yes & \\
Type of resection & $618(64.9)$ \\
Minor & $334(35.1)$ \\
Major & $5(3.0-8.5)$ \\
Tumor size, largest cm & \\
[median (IQR)] & \\
Grade & $753(79.9)$ \\
Well to moderate & $189(20.1)$ \\
Poor to undifferentiated & \\
Lymphovascular invasion & $536(60.8)$ \\
No & $346(39.2)$ \\
Yes & \\
Liver capsule involvement & $491(67.2)$ \\
No & $240(32.8)$ \\
Yes & \\
Margin status & $825(86.8)$ \\
R0 & $104(10.9)$ \\
R1 & $21(2.3)$ \\
R2 & $5.1(3.4-8.8)$ \\
Pathologic TBS & $5.1(3.3-8.5)$ \\
Radiologic TBS &
\end{tabular}

Data are expressed as $n(\%)$ unless otherwise specified

$I Q R$ interquartile range, ASA-PS American Society of Anesthesiologists performance score, $H B V$ hepatitis B virus, $H C V$ hepatitis $\mathrm{C}$ virus, $A S T$ aspartate aminotransferase, $A L T$ alanine aminotransferase, $A F P \alpha$-fetoprotein, PT/INR prothrombin time/international normalized ratio, $B C L C$ Barcelona Clinic Liver Cancer, TBS tumor burden score

algorithm as being the best predictor of OS. Specifically, patients with radiologic TBS $<7.87$ had a 5-year OS of $60.5 \%$ versus only $28.9 \%$ among patients with TBS $>7.87$ (Fig. 1). The model performed well in both the training (cindex 0.641) and validation datasets with bootstrapping resamples (c-index 0.604).

\section{Postoperative CART Model: Factors Associated with Overall Survival (OS) Following Resection}

The postoperative CART model revealed lymphovascular invasion as the best postoperative predictor of OS among BCLC-0/A patients, followed by preoperative AFP levels, ASA class, and CCS. In contrast, among BCLC-B patients, pathologic TBS remained the best predictor of long-term outcomes (Fig. 2). Among BCLC-0/A patients, 5 -year OS was $54.6 \%$ versus $70 \%$ for individuals who did 


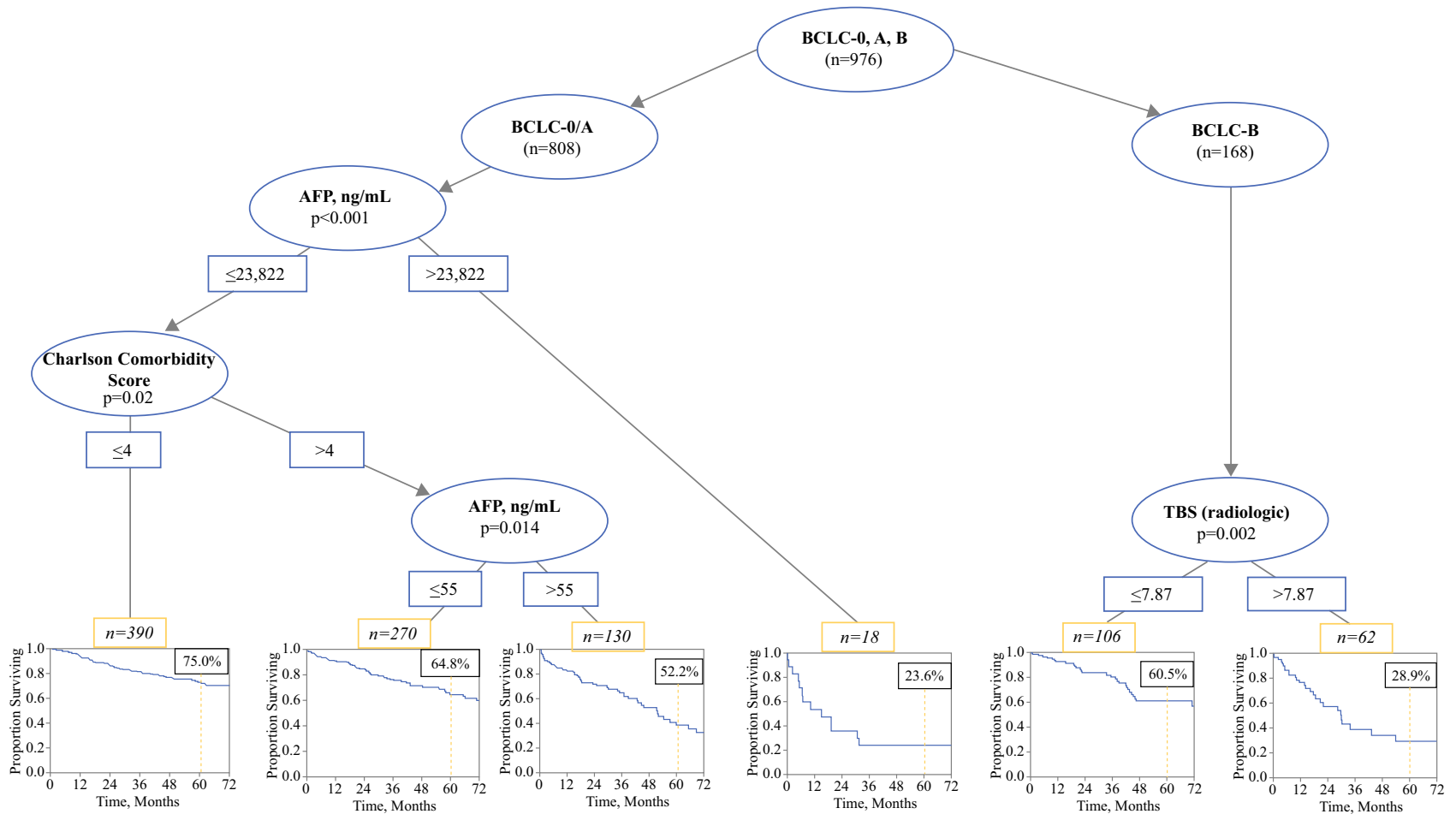

FIG. 1 A CART model depicting the hierarchical association of preoperative factors relative to 5-year overall survival stratified by BCLC stage. $C A R T$ Classification and Regression Tree, BCLC Barcelona Clinic Liver Cancer, AFP $\alpha$-fetoprotein, $T B S$ tumor burden score

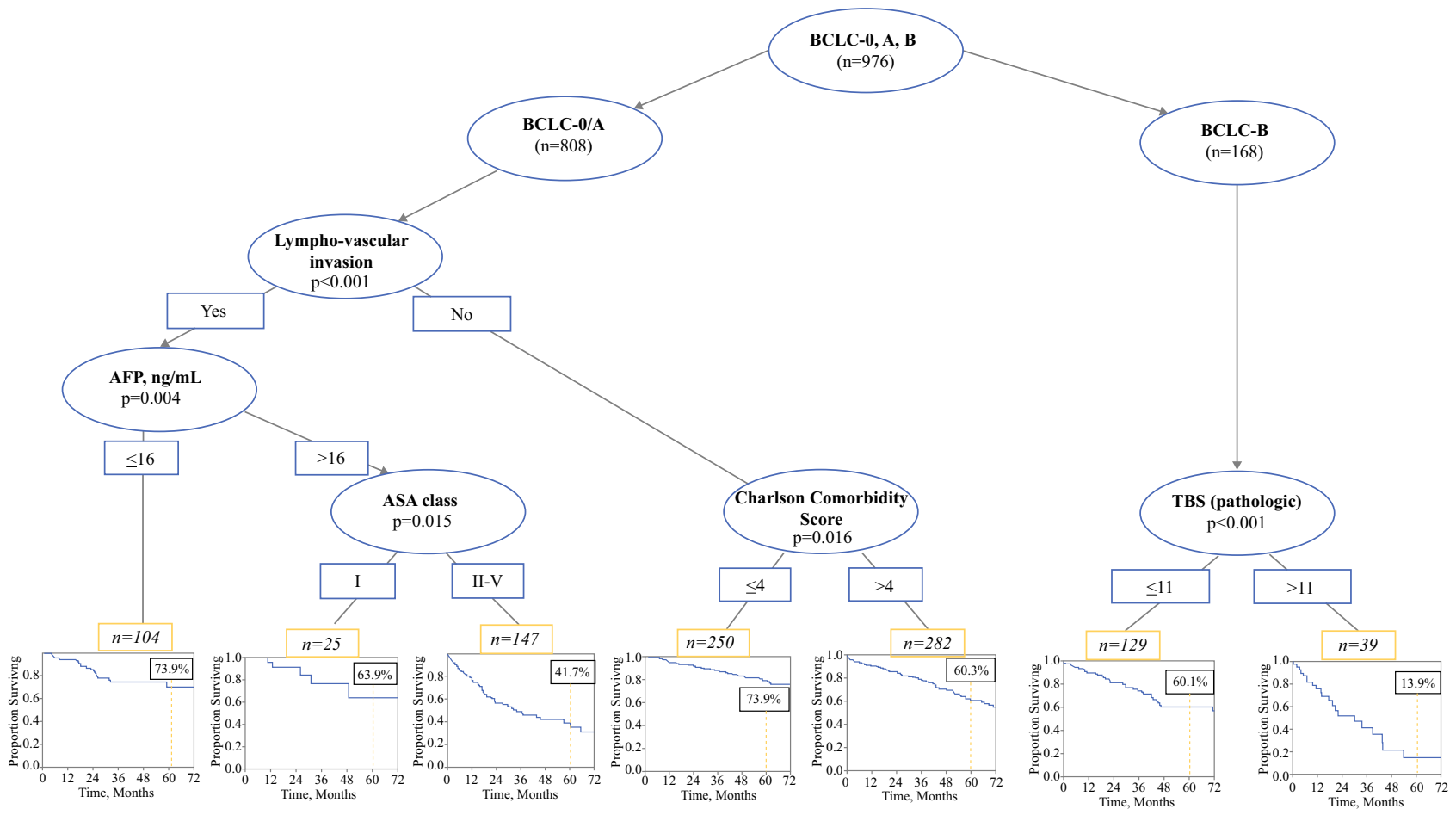

FIG. 2 A CART model depicting the hierarchical association of postoperative factors relative to 5 -year overall survival stratified by BCLC stage. CART Classification and Regression Tree, BCLC
Barcelona Clinic Liver Cancer, ASA American Society of Anesthesiologists, TBS tumor burden score 
and did not have lymphovascular invasion on pathology, respectively $(p<0.001)$ [electronic supplementary Figure 2]. Among individuals with lymphovascular invasion, individuals with the lowest AFP levels $(\leq 16 \mathrm{ng} / \mathrm{mL})$ achieved a 5-year OS as high as $73.9 \%$, whereas among patients with higher AFP levels ( $>16 \mathrm{ng} / \mathrm{mL}$ ), 5-year OS ranged from $41.7 \%$ to $63.9 \%$ depending on the preoperative ASA class. Among patients without lymphovascular invasion on pathology, individuals with CCS $\leq 4$ had a 5-year OS as high as $73.9 \%$, whereas patients with $\mathrm{CCS}>4$ had a 5 -year OS of $60.3 \%$. Of note, among BCLC-B patients, individuals with pathologic TBS $\leq 11$ had a 5-year OS of $60.1 \%$ versus only $13.9 \%$ among patients with TBS $>11$. The prognostic model performed well in both the training (c-index 0.661) and internal bootstrapping validation (c-index 0.634$)$ datasets.

After a median follow-up of 26.5 months (IQR 12.5-51.1), 369 (45.7\%) and 105 (62.5\%) patients had a recurrence following resection of BCLC 0/A and BCLC-B $\mathrm{HCC}$, respectively. Of note, treatment modalities following recurrence were comparable among BCLC-0/A and BCLC B patients (BCLC-0/A vs. BCLC B, repeat hepatectomy: 44/336 [13.1\%] vs. 9/104 [8.7\%]; local ablation: 110/336 [32.7\%] vs. 37/104 [35.6\%]; transarterial embolization/ TACE: $92 / 336$ [27.4\%] vs. $31 / 104$ [29.8\%]; sorafenib or other supportive therapy: $90 / 336[n=26.8 \%]$ vs. $27 / 104$ [26.0\%]; $p=0.64)$.

\section{Multivariable Analysis of OS}

Several factors were associated with prognosis following resection for BCLC-0/A and BCLC-B HCC (electronic supplementary Table 1). On multivariable analysis, after adjusting for competing risk factors, age $>65$ years (hazard ratio [HR] 1.36, 95\% confidence interval [CI] 1.03-1.80; $p=0.03), \quad \mathrm{CCS}>4 \quad(\mathrm{HR} 1.77, \quad 95 \% \quad$ CI $1.33-2.34$; $p<0.001), \quad$ AFP $>400 \mathrm{ng} / \mathrm{mL} \quad(\mathrm{HR} \quad 1.41, \quad 95 \% \quad$ CI $1.03-1.94 ; p=0.033$ ), lymphovascular involvement (HR $1.70,95 \%$ CI $1.26-2.29 ; p<0.001$ ), R1/R2 resection (HR $1.54,95 \%$ CI $1.03-2.29 ; p=0.036$ ), and pathologic TBS (HR 1.04, 95\% CI 1.02-1.07; $p=0.001$ ) were independent predictors of OS among BCLC-0/A patients. In contrast, only poor/undifferentiated tumor grade (HR $2.43,95 \%$ CI 1.37-4.30; $p=0.002)$ and pathologic TBS (HR 1.13, 95\% CI 1.06-1.19; $p<0.001)$ independently predicted worse OS among BCLC-B patients undergoing resection (Table 2).

\section{DISCUSSION}

Over the last three decades, the incidence of HCC has been steadily increasing in both Eastern and Western countries with a concomitant increase in HCC mortality. ${ }^{1,15}$ Accurate preoperative staging is critical to define the prognosis of patients with $\mathrm{HCC}$ and inform treatment recommendations. ${ }^{3,4}$ Among the traditional staging systems, the BCLC classification-largely adopted by physicians in the West-not only assesses patient prognosis but also assigns treatment allocation based on prognostic subclasses. ${ }^{5,6}$ Although endorsed by the EASL and the American Association for the Study of Liver Disease (AASLD) ${ }^{5-7}$ this system has recently been questioned relative to the proposed treatment allocation. Indeed, a number of investigators have reported favorable outcomes following resection beyond the current criteria (i.e. BCLC$\mathrm{B}$ or even BCLC-C HCC). Nevertheless, there is currently no consensus as to which patients will benefit the most from surgery. As such, stratifying patients into distinct prognostic groups within each BCLC stage has been a topic of debate. The current study was important because we utilized a CART machine-based learning model to identify the most predictive factors relative to long-term survival among patients undergoing resection within (i.e. BCLC-0/ A) and beyond (i.e. BCLC-B) the current BCLC resection criteria. Using this technique, we were able to define six prognostic groups of patients based on factors demonstrated to be the most predictive of OS. Of note, AFP and CCS dominated prognosis for BCLC-0/A patients (within BCLC), whereas radiologic TBS predicted OS the most for BCLC-B patients (beyond BCLC). TBS was consistently identified as the most important prognostic factor among BCLC-B patients, either pre- (radiologic TBS) or postoperatively (pathologic TBS). In contrast, lymphovascular invasion, followed by AFP, CCS, and ASA class mostly determined OS in the postoperative setting for BCLC-0/A patients. In addition, 5-year OS ranged from $23.6 \%$ to $75 \%$ among different BCLC-0/A subgroups, whereas BCLC-B subgroups had a 5-year OS ranging from $28.9 \%$ to $60.5 \%$. To the best of our knowledge, this is the first study in the literature to utilize a machine learning method as a means to identify different prognostic groups within BCLC stages in the pre- and postoperative setting.

Several investigators have suggested that the BCLC classification may be an oversimplification of how physicians treat HCC patients. Indeed, a number of studies have demonstrated heterogeneous outcomes after resection of HCC, even within the same BCLC stage. ${ }^{11,16}$ To this point, our own group reported different outcomes among patients within the BCLC-A stage that were largely dependent on tumor size. ${ }^{17}$ Indeed, patients with single large tumors $(>5 \mathrm{~cm}$ ) had a 5 -year OS of $56.9 \%$, which was markedly lower than the OS among patients with smaller size solitary tumors (5-year OS: 69\%), yet comparable with patients who underwent surgery for BCLC-B HCC (5-year OS: $49.9 \% ; p=0.259) .{ }^{17}$ In addition, Wada and colleagues reported a distinct prognosis for patients undergoing 
TABLE 2 Multivariable analysis of survival ${ }^{\mathrm{a}}$

\begin{tabular}{|c|c|c|c|c|c|c|}
\hline \multirow[t]{2}{*}{ Variable } & \multicolumn{3}{|c|}{ BCLC-0/A } & \multicolumn{3}{|c|}{ BCLC-B } \\
\hline & HR & $95 \% \mathrm{CI}$ & $p$ value & HR & $95 \% \mathrm{CI}$ & $p$ value \\
\hline \multicolumn{7}{|l|}{ Age, years } \\
\hline$\leq 65$ & Ref & & & & & \\
\hline$>65$ & 1.36 & $1.03-1.80$ & $\mathbf{0 . 0 3}$ & - & & \\
\hline \multicolumn{7}{|l|}{$\mathrm{CCS}$} \\
\hline$\leq 4$ & Ref & & & & & \\
\hline$>4$ & 1.77 & $1.33-2.34$ & $<\mathbf{0 . 0 0 1}$ & - & & \\
\hline \multicolumn{7}{|l|}{$\mathrm{AFP}, \mathrm{ng} / \mathrm{mL}$} \\
\hline$\leq 400$ & Ref & & & & & \\
\hline$>400$ & 1.41 & $1.03-1.94$ & $\mathbf{0 . 0 3 3}$ & - & & \\
\hline \multicolumn{7}{|l|}{ Grade } \\
\hline Well/moderate & Ref & & & Ref & & \\
\hline Poor/undifferentiated & 1.33 & $0.98-1.81$ & 0.069 & 2.43 & $1.37-4.30$ & 0.002 \\
\hline \multicolumn{7}{|c|}{ Lymphovascular involvement } \\
\hline No & Ref & & & - & & \\
\hline Yes & 1.70 & $1.26-2.29$ & $<\mathbf{0 . 0 0 1}$ & & & \\
\hline \multicolumn{7}{|l|}{ Margin } \\
\hline R0 & Ref & & & Ref & & \\
\hline $\mathrm{R} 1 / \mathrm{R} 2$ & 1.54 & $1.03-2.29$ & 0.036 & 1.70 & $0.92-3.16$ & 0.093 \\
\hline Pathologic TBS & 1.04 & $1.02-1.07$ & 0.001 & 1.13 & $1.06-1.19$ & $<\mathbf{0 . 0 0 1}$ \\
\hline
\end{tabular}

$C C S$ Charlson comorbidity score, $A F P$-fetoprotein, BCLC Barcelona Clinic Liver Cancer, TBS tumor burden score, $H R$ hazard ratio, $C I$ confidence interval, Ref reference

${ }^{\mathrm{a}}$ The final step of the backward stepwise model is presented for both BCLC-0/A and B stages

resection for multinodular (BCLC-B) HCC, thus highlighting the need for further subclassification of BCLC stages, especially BCLC-B tumors. ${ }^{10}$ Ruan et al. recently proposed a nomogram based on total tumor volume, ChildPugh class, plasma fibrinogen levels, and the presence of portal vein thrombosis to predict OS prior to resection for HCC beyond BCLC-A stage. ${ }^{18}$ The authors suggested three groups of patients (i.e. low, medium, and high risk) with the lowest risk group having a 5-year OS and RFS similar to that of the BCLC-A patients (5-year OS: $83.1 \%$ vs. $83.1 \%, p=0.46$; 5 -year RFS: $50.6 \%$ vs. $55.9 \%$, $p=0.68) .{ }^{18}$ The current study revealed that TBS was associated with OS in both BCLC-0/A and BCLC-B patients, even after adjusting for competing risk factors. Of note, for each point increase in TBS, the hazards of death increased by $4 \%$ among BCLC-0/A patients, whereas the corresponding increase was $13 \%$ among BCLC-B patients (Table 2). These data were consistent with a recent study by the ITA.LI.CA group that demonstrated a $6 \%$ increased risk of death for each point increase in TBS. ${ }^{13,19}$ Of note, in the current study, both pre- and postoperative CART models demonstrated that both radiologic and pathologic TBS were the most important indicators of long-term outcomes among BCLC-B patients undergoing resection for HCC. Importantly, surgery may offer patients with a radiologic $\mathrm{TBS} \leq 7.8$ an acceptable 5-year OS (60.5\%), which was even better than that of certain groups of patients presenting with BCLC-0/A tumors (Fig. 1). As such, by calculating radiologic TBS, clinicians could preoperatively identify patients with tumors beyond the current BCLC resection guidelines who are most likely to derive the most benefit from surgery. In addition, pathologic TBS should be considered an important predictor of survival following resection for HCC beyond the current BCLC resection guidelines.

Recently, an increasing number of studies have reported acceptable outcomes following resection for BCLC-B HCC, suggesting that liver resection may be justified in select patients beyond the BCLC resection criteria. $^{20-22}$ A recent study reported a 5-year OS of $63.4 \%$ following liver resection for multinodular BCLCB HCC. ${ }^{10}$ Furthermore, in a propensity-matched cohort, Kim et al. reported superior outcomes for patients with BCLC-B who were treated with resection versus nonsurgical treatment (5-year OS: $63 \%$ vs. $22 \%$ ). ${ }^{20}$ In addition, a recent meta-analysis demonstrated a survival 
benefit for surgery compared with TACE among BCLC$\mathrm{B}$ patients, thus questioning the treatment algorithm proposed by the current BCLC classification schema. ${ }^{22}$ By analyzing a large multi-institutional database, we herein reported a 5-year OS of 50.2\% among BCLC-B patients. Interestingly, BCLC-B patients with radiologic TBS $\leq 7.87$ had a 5 -year OS as high as $60.5 \%$, which was even higher than the OS of BCLC-A patients with extremely high AFP (5-year OS: $23.6 \%$ ) or AFP $>55$ $\mathrm{ng} / \mathrm{mL}$, and CCS $>4$ (5-year OS: $52.2 \%)$. As such, the current study suggests that surgery may indeed be beneficial in select patients with BCLC-B HCC, especially when TBS is low.

The present study should be interpreted in the light of certain limitations. The retrospective nature of the study may have introduced a selection bias as to which patients were offered surgery (i.e. patients with BCLC-B HCC possibly had more favorable tumor biology). In addition, the vast majority of patients included in the study had favorable liver function (i.e. Child-Pugh class A), and thus the findings may not pertain to patients with more severe underlying liver disease. Furthermore, the current study did not include patients with BCLC-C tumors. As such, future studies should aim to verify the results in these patient populations. Although prophylactic treatment after surgery may be beneficial in certain circumstances (i.e. presence of microvascular invasion, tumor size $>5 \mathrm{~cm}),{ }^{23,24}$ this information was not available in the current dataset, although the overall use of prophylactic treatment was presumably very low. Finally, although the machine learning techniques, such as the CART models, improve our ability to preoperatively predict patient prognosis, incorporation of genetic and molecular profiles of HCC will be necessary to further optimize the predictive ability of these models.

\section{CONCLUSIONS}

Surgery provided acceptable outcomes among select patients with BCLC-B HCC. While AFP and CCS appeared to be the best prognostic factors associated with survival among BCLC-0/A patients, a machine-based CART model identified TBS (either radiologic or pathologic) as the best predictor of outcomes among BCLC-B patients undergoing resection. These data further emphasize the need for refinement of the proposed BCLC treatment algorithm and advocate for the use of TBS for risk stratification and prognostic estimation among patients presenting with HCC beyond the current resection criteria.

\section{ACKNOWLEDGEMENT None}

FUNDING No sources of funding were used to assist in the preparation of this study.

DISCLOSURE The authors have no conflicts of interest to disclose.

\section{REFERENCES}

1. Fujiwara N, Friedman SL, Goossens N, Hoshida Y. Risk factors and prevention of hepatocellular carcinoma in the era of precision medicine. J Hepatol. 2018;68(3):526-49.

2. Petrick JL, Kelly SP, Altekruse SF, McGlynn KA, Rosenberg PS. Future of Hepatocellular Carcinoma Incidence in the United States Forecast Through 2030. Journal of clinical oncology. 2016;34(15):1787-94.

3. Marrero JA, Fontana RJ, Barrat A, et al. Prognosis of hepatocellular carcinoma: comparison of 7 staging systems in an American cohort. Hepatology. 2005;41(4):707-16.

4. O'Neil BH, Venook AP. Hepatocellular carcinoma: the role of the North American GI Steering Committee Hepatobiliary Task Force and the advent of effective drug therapy. Oncologist. 2007;12(12):1425-32.

5. Cillo U, Vitale A, Grigoletto F, et al. Prospective validation of the Barcelona Clinic Liver Cancer staging system. J Hepatol. 2006;44(4):723-31.

6. European Association for the Study of the Liver. EASL Clinical Practice Guidelines: Management of hepatocellular carcinoma. $J$ Hepatol. 2018;69(1):182-236.

7. Marrero JA, Kulik LM, Sirlin CB, et al. Diagnosis, Staging, and Management of Hepatocellular Carcinoma: 2018 Practice Guidance by the American Association for the Study of Liver Diseases. Hepatology. 2018;68(2):723-50.

8. Torzilli G, Belghiti J, Kokudo N, et al. A snapshot of the effective indications and results of surgery for hepatocellular carcinoma in tertiary referral centers: is it adherent to the EASL/AASLD recommendations? An observational study of the HCC East-West study group. Ann Surg. 2013;257(5):929-37.

9. McCormack L, Petrowsky H, Clavien PA. Surgical therapy of hepatocellular carcinoma. Eur $J$ Gastroenterol Hepatol. 2005;17(5):497-503.

10. Wada H, Eguchi H, Noda T, et al. Selection criteria for hepatic resection in intermediate-stage (BCLC stage B) multiple hepatocellular carcinoma. Surgery. 2016;160(5):1227-35.

11. Guo H, Wu T, Lu Q, et al. Surgical resection improves long-term survival of patients with hepatocellular carcinoma across different Barcelona Clinic Liver Cancer stages. Cancer Manag Res. 2018;10:361-69.

12. Strasberg SM. Nomenclature of hepatic anatomy and resections: a review of the Brisbane 2000 system. $J$ Hepatobiliary Pancreat Surg. 2005;12(5):351-5.

13. Sasaki K, Morioka D, Conci S, et al. The Tumor Burden Score: A New "Metro-ticket" Prognostic Tool For Colorectal Liver Metastases Based on Tumor Size and Number of Tumors. Ann Surg. 2018;267(1):132-41.

14. Breiman L. Classification and regression trees. Belmont, CA: Wadsworth International Group; 1984.

15. Beal EW, Tumin D, Kabir A, et al. Trends in the Mortality of Hepatocellular Carcinoma in the United States. J Gastrointest Surg. 2017;21(12):2033-38.

16. Cho Y, Sinn DH, Yu SJ, et al. Survival Analysis of Single Large $(>5 \mathrm{~cm})$ Hepatocellular Carcinoma Patients: BCLC A versus B. PLoS One. 2016;11(11):e0165722.

17. Tsilimigras DI, Bagante F, Sahara K, et al. Prognosis after Resection for Barcelona Clinic Liver Cancer (BCLC) Stage 0, A 
and B Hepatocellular Carcinoma: A Comprehensive Assessment of the Current BCLC Classification. Ann Surg Oncol. 2019;26(11):3693-700.

18. Ruan DY, Lin ZX, Wang TT, et al. Nomogram for preoperative estimation of long-term survival of patients who underwent curative resection with hepatocellular carcinoma beyond Barcelona clinic liver cancer stage A1. Oncotarget. 2016;7(38):61378-89.

19. Vitale A, Lai Q, Farinati F, et al. Utility of Tumor Burden Score to Stratify Prognosis of Patients with Hepatocellular Cancer: Results of 4759 Cases from ITA.LI.CA Study Group. J Gastrointest Surg. 2018;22(5):859-71.

20. Kim H, Ahn SW, Hong SK, et al. Survival benefit of liver resection for Barcelona Clinic Liver Cancer stage B hepatocellular carcinoma. Br J Surg. 2017;104(8):1045-52.

21. Liang L, Xing $H$, Zhang $H$, et al. Surgical resection versus transarterial chemoembolization for BCLC intermediate stage hepatocellular carcinoma: a systematic review and meta-analysis. HPB (Oxford). 2018;20(2):110-9.

22. Hyun MH, Lee YS, Kim JH, et al. Hepatic resection compared to chemoembolization in intermediate- to advanced-stage hepatocellular carcinoma: a meta-analysis of high-quality studies. Hepatology. 2018;68(3):977-93.

23. He S, Fan X, Ma H, et al. Effect of prophylactic TACE on 5-year survival of patients with hepatocellular carcinoma after hepatectomy. Oncol Lett. 2019;18(2):1824-30.

24. Huang SX, Wu YL, Tang CW, et al. Prophylactic hepatic artery infusion chemotherapy improved survival after curative resection in patients with hepatocellular carcinoma. Hepatogastroenterology. 2015;62(137):122-5.

Publisher's Note Springer Nature remains neutral with regard to jurisdictional claims in published maps and institutional affiliations. 